\title{
Helfrich free energy for aggregation and adhesion
}

Cite as: J. Chem. Phys. 110, 3148 (1999); https://doi.org/10.1063/1.478190

Submitted: 01 April 1998 . Accepted: 06 November 1998. Published Online: 29 January 1999

E. M. Blokhuis, and W. F. C. Sager

\section{ARTICLES YOU MAY BE INTERESTED IN}

Spontaneous curvature of bilayer membranes from molecular simulations: Asymmetric lipid densities and asymmetric adsorption

The Journal of Chemical Physics 142, 054101 (2015); https://doi.org/10.1063/1.4906149

Thermodynamic expressions for the Tolman length

The Journal of Chemical Physics 124, 074701 (2006); https://doi.org/10.1063/1.2167642

The Effect of Droplet Size on Surface Tension

The Journal of Chemical Physics 17, 333 (1949); https://doi.org/10.1063/1.1747247

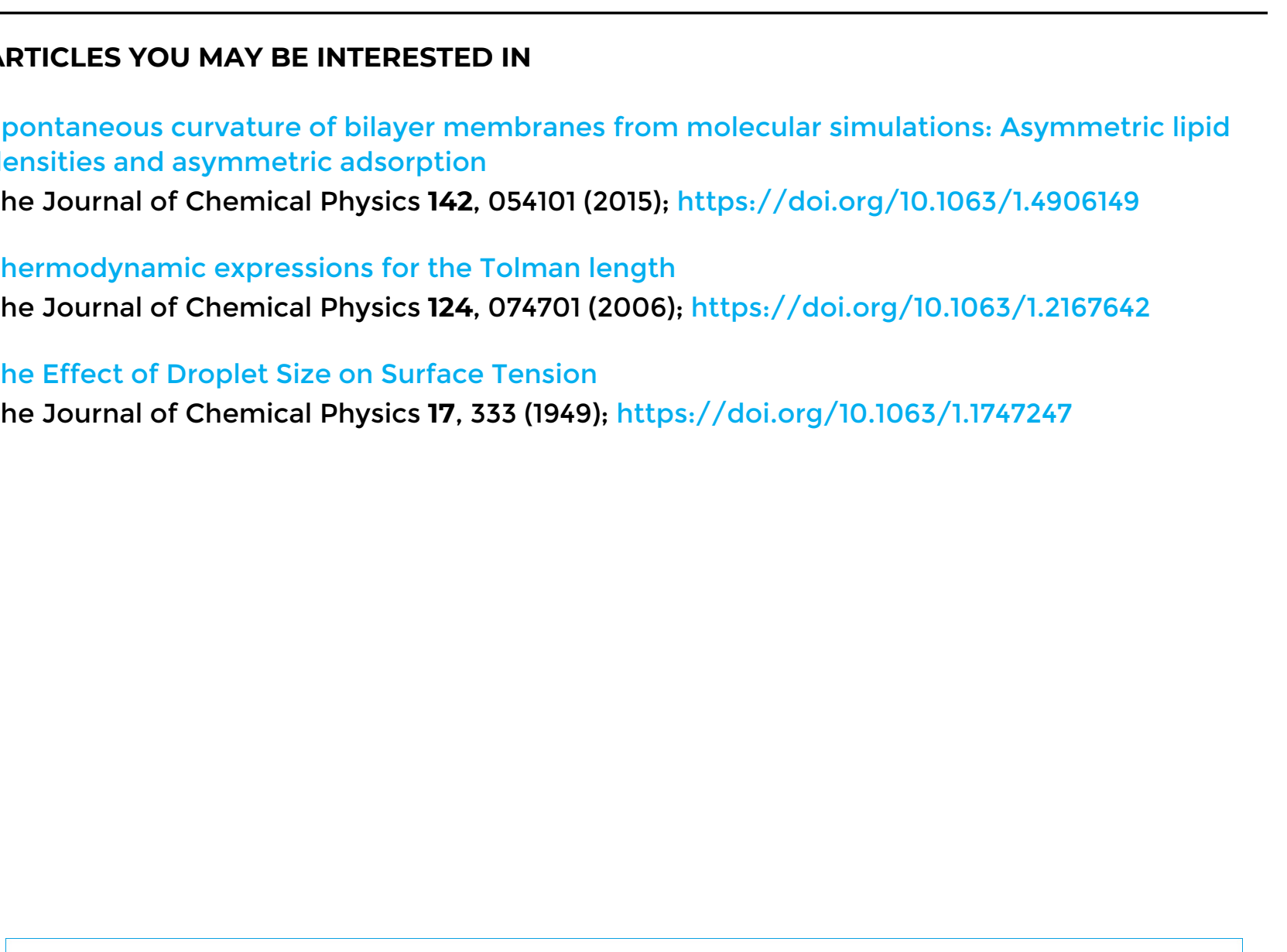




\title{
Helfrich free energy for aggregation and adhesion
}

\author{
E. M. Blokhuis and W. F. C. Sager ${ }^{\mathrm{a})}$ \\ Department of Physical and Macromolecular Chemistry, Leiden Institute of Chemistry, P.O. Box 9502, \\ 2300 RA Leiden, The Netherlands
}

(Received 1 April 1998; accepted 6 November 1998)

\begin{abstract}
We present a theoretical study of the shape and free energy of a vesicle (or microemulsion droplet) adhered to a substrate (other droplet) based on the expression for the surface free energy by Helfrich. Analytical formulas are presented for the shape and free energy when the rigidity constant for bending, $k$, is small; i.e., when $(k / \sigma)^{1 / 2}$, with $\sigma$ the surface tension, is small compared to the typical dimension of the vesicle $(k / \sigma)^{1 / 2} \ll V^{1 / 3}$, with $V$ the vesicle volume. These formulas are compared with numerical solutions of the shape equations such as those first provided in the work by Seifert and Lipowsky. Results are presented when the exact formulas are applied to study the onset of microemulsion droplet aggregation, e.g., dimer formation, in terms of the usual coefficients in the Helfrich free energy expression, such as the rigidity constant for bending and the spontaneous curvature. (C) 1999 American Institute of Physics. [S0021-9606(99)52406-9]
\end{abstract}

The Helfrich expression for the surface free energy ${ }^{1}$ has been successfully applied to describe the shape and free energy of membranes, vesicles, microemulsion droplets $^{2}$ or even fluctuations of the simple liquid-vapor interface. ${ }^{3}$ It describes the free energy for bending the surface, complementing the usual surface tension energy for extending the surface, in terms of two elasticity or rigidity constants, $k$ and $\bar{k}$. Seifert et $_{\text {al. }}{ }^{4,5}$ were the first to apply the Helfrich free energy to describe adhesion. They calculated the shape and free energy of a vesicle adhered to a solid substrate (see Fig. 1a). Unfortunately the differential equations derived from the minimization of the Helfrich free energy cannot, in general, be solved analytically so that Seifert et al. had to resort to solving these shape equations numerically. However, the large number of parameters such as the prescribed surface area $A$, vesicle volume $V$, the rigidity constants, as well as the adhesion energy makes numerical work tedious and the need arises for limiting analytical results. In this Communication we present such a limiting solution by calculating the shape and free energy of a vesicle adhered to a substrate under the condition that the rigidity constant $k$ is small. Specifically, the length constructed from the rigidity constant and surface tension $\sigma$ must be small compared to the typical dimension of the system, $(k / \sigma)^{1 / 2} \ll V^{1 / 3}$.

Our formulas can also be applied to study the onset of droplet aggregation in microemulsions. In this case, instead of a vesicle adhered to a substrate one describes the adhesion of two microemulsion droplets forming a dimer (see Fig. 1b). In the common description of aggregation of microemulsion droplets the analogy with liquid state theories has been used treating the microemulsion droplets as hard spheres or sticky hard spheres. ${ }^{6,7}$ The phase separation described in terms of a liquid-gas transition is to be contrasted with another approach using the Helfrich free energy, originally applied to

\footnotetext{
a) Present address: Faculty of Chemical Technology, Membrane Technology, University of Twente, P.O. Box 217, 7500 AE Enschede, The Netherlands.
}

describe the nonspherical shapes of membranes and vesicles, ${ }^{8}$ to understand microemulsion phase diagrams and to calculate form fluctuations and polydispersity for microemulsion droplets. ${ }^{9,10}$

Experiments have shown ${ }^{11}$ that aggregation processes in microemulsions occur both with increasing and decreasing temperature depending on the microemulsion system studied (e.g., ionic or nonionic surfactant, water-in-oil or oil-in-water microemulsion). In both cases, however, aggregation processes take place in the direction of vanishing spontaneous curvature (e.g., with increasing temperature in ionic, waterin-oil microemulsions) eventually leading to structural changes such as cylinder or channel formation. It thus seems natural to apply the Helfrich free energy to the onset of microemulsion aggregation by calculating, as is done in this Communication, the change in shape and free energy when two microemulsion droplets form a dimer. In this way the "stickiness"-parameter",12 from the sticky hard-spheremodel can be expressed in terms of the spontaneous curvature and rigidity constants.

Although the analysis is thus more generally applicable, we first focus on the specific problem of a vesicle adhered to a solid substrate (Fig. 1a). We assume that the range of the interaction between the substrate and the vesicle is much smaller than the typical dimension of the vesicle. In fact, the interaction will be approximated by a delta function located at the substrate. Our analysis is thus equivalent to a more general one in which the surface tension is assumed to be different on part of the closed surface. This might be due to the presence of a substrate (adhesion) or another closed surface (particle aggregation) but might also be due to some external force or boundary conditions (DOC model, ${ }^{13}$ see also Ref. 14), chemical modification of part of the surface, surface pinching, ${ }^{15}$ etc.

The Helfrich form for the surface free energy of a (weakly) curved interface introduces the Tolman length $\delta$ (Ref. 16) [related to the radius of spontaneous curvature $R_{0}$ by $\delta \sigma=2 k / R_{0}$ (Ref. 3)], the rigidity constant associated with 


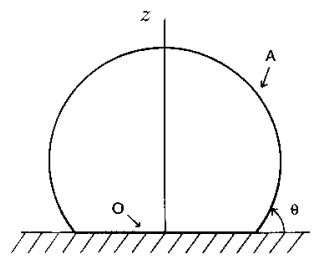

(a) (b)
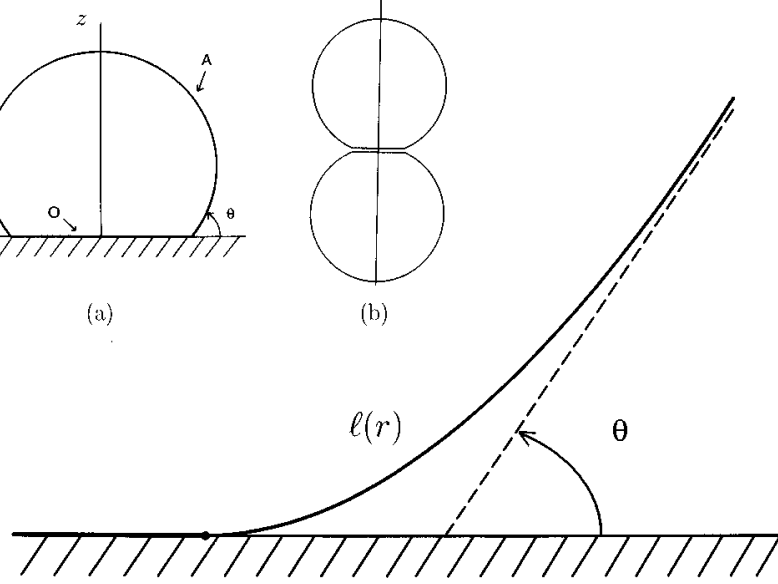

FIG. 1. Height profile $\ell(r)$ with $r$ the radial distance to the $z$-axis. Lengths are in units of $(k / \sigma)^{1 / 2}$. The dashed line is the asymptotic spherical-capprofile which meets the substrate with contact angle $\theta$. See also inset (a): vesicle adhered to a substrate located at $z=0 ; A$ is the surface area of the whole droplet including the surface area $O$ that is in contact with the substrate. Inset (b): two aggregated microemulsion droplets.

bending, $k$, and the rigidity constant associated with Gaussian curvature, $\bar{k}$,

$$
F_{H}=\int d A\left[\sigma-\delta \sigma J+\frac{k}{2} J^{2}+\bar{k} K\right],
$$

where $\sigma$ is the surface tension of the planar surface. The above form for the free energy is the most general form in an expansion up to second order in curvature, and can be viewed as defining the four coefficients $\sigma, \delta, k$ and $\bar{k}$. It features an integral over the whole surface area, $A$, of the total curvature, $J=1 / R_{1}+1 / R_{2}$ and Gaussian curvature, $K$ $=1 /\left(R_{1} R_{2}\right)$ with $R_{1}$ and $R_{2}$ the principal radii of curvature at a certain point on the surface $A$. The rigidity constant associated with Gaussian curvature $\bar{k}$ is a measure of the energy cost for topological changes of the surface. In our case the topology is fixed and the term proportional to $\bar{k}$ is dropped. To the above free energy we add a term describing the contact energy with the substrate ${ }^{4}$

$$
F_{s}=\int d O[\Delta \sigma-\sigma],
$$

where $O$ is the area of substrate-vesicle contact and where $\Delta \sigma \equiv \sigma_{s v}-\sigma_{s}$ is the difference in surface tension of the substrate-vesicle surface and the bare substrate. Since the integration in Eq. (1) is over the whole surface area $A$ (including $O$ ), we need to subtract $\sigma$ from $\Delta \sigma$ in the equation above. In the case of two microemulsion droplets forming a dimer $\Delta \sigma=\sigma_{b} / 2$, with $\sigma_{b}$ the surface tension of the bilayer formed in between the two droplets. The surface tension of the bilayer or, in fact, the complete interaction energy between the surfactant monolayers as a function of separation distance, is, in principle, experimentally accessible by surface force apparatus (SFA) measurements. ${ }^{17}$ Recently Fletcher and Petsev ${ }^{18}$ considered the effect of the full interaction potential (van der Waals), including increased droplet deformability with vanishing spontaneous curvature, on the aggregation of microemulsion droplets.
The total free energy to be minimized with respect to the shape of the vesicle is the sum of the curvature free energy Eq. (1), the substrate interaction energy Eq. (2), and $-\Delta p V$, where $\Delta p$ equals the pressure difference between the inside and the outside of the vesicle: $\Omega=F_{H}+F_{s}$ $-\Delta p V$. In the minimization of $\Omega$, one can view $\Delta p$ as either the given pressure difference or as the Lagrange multiplier fixing the volume of the vesicle. In the latter case the free energy that is minimized is $F=F_{H}+F_{s}$, rather than $\Omega$, with $-\Delta p V$ added to $F$ to fix the volume. Similarly one can view the surface tension $\sigma$ as it appears in Eq. (1) as the macroscopically given surface tension or as the Lagrange multiplier fixing the total surface area $A$ of the vesicle. In the case of a vesicle adhered to a substrate, the most appropriate ensemble is that of constant volume and constant surface area, ${ }^{8}$ while in a system of aggregating microemulsion droplets the total volume is fixed in the one-phase region, while $\Delta p$ is fixed $(\Delta p=0)$ in the two-phase region, where the microemulsion phase coexists with an excess water or oil phase. ${ }^{9,19}$ We stress, however, that in all these ensembles the free energy to be minimized has the form of $\Omega$ with the free energy corresponding to these different ensembles derived from $\Omega$ by making the appropriate changes.

Below we first present the calculation for the case that $k=0$, i.e., taking the expansion in Eq. (1) only to first order in the curvature, followed by the calculation for $k \neq 0$ but small, $(k / \sigma)^{1 / 2} \ll V^{1 / 3}$.

It follows from the shape equations that when $k=0$, the shape of the nonattached part of the vesicle is that of a spherical cap with radius $R$ (inset (a) Fig. 1). Integration of the free energy over the surface areas $A$ and $O$ in Eqs. (1) and (2) can then directly be carried out. In doing so one has to take care of a contribution proportional to $\delta$ arising from the integration of the total curvature $J$ across the kink in the profile where the spherical part of the vesicle meets the substrate. This implies that it is not correct to subdivide the integration over $A$ into an integration over the spherical part and the flat part $(O)$. The resulting free energy is now solely expressed in terms of the radius $R$ and contact angle $\theta$ :

$$
\begin{aligned}
\Omega_{0}(R, x)= & 2 \pi \sigma R^{2}(1+x)+\pi \Delta \sigma R^{2}\left(1-x^{2}\right) \\
& -4 \pi \delta \sigma R(1+x)-2 \pi \delta \sigma R\left(1-x^{2}\right)^{1 / 2} \arccos (x) \\
& -\Delta p(\pi / 3) R^{3}\left(2+3 x-x^{3}\right),
\end{aligned}
$$

where we have defined $x \equiv \cos \theta$ and where the subscript 0 to the free energy denotes that we have taken $k=0$. Finally, $R$ and $x$ are determined by minimizing $\Omega_{0}(R, x)$ with respect to $R$ and $x$. One finds

$$
\begin{aligned}
& \Delta p=\frac{2 \sigma}{R}\left(1-\frac{\delta}{R}\right) \\
& \Delta \sigma=\sigma x-\frac{\delta \sigma}{R} x+\frac{\delta \sigma}{R} \frac{\arccos (x)}{\left(1-x^{2}\right)^{1 / 2}} .
\end{aligned}
$$

The first equation is the well-known Laplace equation with the Tolman correction. In fact, the Tolman length is usually defined by the above equation. ${ }^{16}$ The second equation determines the value of the contact angle. It reduces to Young's equation $^{20} \sigma_{s v}=\sigma_{s}+\sigma \cos \theta$ when one inserts $\delta=0$. 
When $k \neq 0$ the shape of the nonattached part of the surface is not necessarily spherical, just as the shape of the free vesicle will generally differ from that of a sphere. ${ }^{8}$ For small $k$ the difference with sphericity is dominantly located near the kink. The underlying physics behind this is the infinite contribution from the kink to the bending energy. The result is that for any finite value of the rigidity constant, the first derivative of the vesicle profile $\ell(r)$, where $r$ is the radial distance (see Fig. 1), must be continuous. Seifert et al. ${ }^{4}$ furthermore showed that the radius of curvature at the substrate is related to the interaction energy with the substrate by $1 / R_{1}=(2(\sigma-\Delta \sigma) / k)^{1 / 2}$. It was also remarked by Seifert et al. ${ }^{4}$ that for small $k$ the typical length scale over which the profile differs from the spherical-cap-profile near the kink is determined by $(k / \sigma)^{1 / 2}$, while the difference of the rest of the profile with the spherical-cap-profile is of $\mathcal{O}\left(k / \sigma R^{2}\right)$. In the following we expand around the spherical-cap-profile neglecting all terms of $\mathcal{O}\left(1 / R^{2}\right)$. Furthermore we assume $\delta \ll R$ treating corrections in $\delta / R$ to the same order as $(k / \sigma)^{1 / 2} / R$.

With the condition that the contact angle is equal to zero, the height profile $\ell(r)$ can be calculated, analytically, from the minimization of the free energy. It is given in terms of the asymptotic contact angle $\theta$ defined by $\ell^{\prime}(r) \rightarrow \tan \theta$, when $r \rightarrow \infty$ (see Fig. 1) and parametrized by the angle $\alpha$ which runs from $0(y \rightarrow \infty)$ to $\theta(y=0)$ :

$$
\ell(y)=\left\{\begin{aligned}
y(\alpha)= & \cos \theta\left[\ln \left(\tan \left(\frac{\theta}{4}\right)\right)-\ln \left(\tan \left(\frac{\alpha}{4}\right)\right)\right] \\
& -2 \cos \left(\theta-\frac{\alpha}{2}\right)+2 \cos \left(\frac{\theta}{2}\right) \\
\ell(\alpha) & =\sin \theta\left[\ln \left(\tan \left(\frac{\theta}{4}\right)\right)-\ln \left(\tan \left(\frac{\alpha}{4}\right)\right)\right] \\
& -2 \sin \left(\theta-\frac{\alpha}{2}\right)+2 \sin \left(\frac{\theta}{2}\right)
\end{aligned}\right.
$$

where $y$ is the radial distance $r$ shifted so that $\ell(y=0)=0$. Furthermore $\ell$ and $y$ are rescaled by $(k / \sigma)^{1 / 2}$. One can show that this profile obeys the relation by Seifert et al. ${ }^{4}$ concerning the radius of curvature at the substrate.

Using this profile one is now able to calculate the leading order correction $\Omega_{1}(R, x)$ to the free energy $\Omega(R, x)$ $=\Omega_{0}(R, x)+\Omega_{1}(R, x)+\mathcal{O}\left(1 / R^{2}\right)$. One finds

$$
\begin{aligned}
\Omega_{1}(R, x)= & 2 \pi R(k \sigma)^{1 / 2} 2^{1 / 2}(1-x)^{1 / 2}\left[2^{3 / 2}(1+x)^{1 / 2}\right. \\
& -(2+x)-\Delta \sigma / \sigma] .
\end{aligned}
$$

It should be noted that the leading order correction to the free energy due to the presence of a finite rigidity thus scales as $k^{1 / 2}$, i.e., the free energy is not analytic in $k$. Minimization of $\Omega_{0}(R, x)+\Omega_{1}(R, x)$ with respect to $R$ and $x$ now yields

$$
\begin{aligned}
\Omega= & (\pi / 3) \sigma R_{p}^{2}\left(2+3 x_{0}-x_{0}^{3}\right)-4 \pi \delta \sigma R_{p}\left(1+x_{0}\right) \\
& -2 \pi \delta \sigma R_{p}\left(1-x_{0}^{2}\right)^{1 / 2} \arccos \left(x_{0}\right) \\
& +4 \pi R_{p}(k \sigma)^{1 / 2}\left(1-x_{0}^{2}\right)^{1 / 2}\left[2-2^{1 / 2}\left(1+x_{0}\right)^{1 / 2}\right],
\end{aligned}
$$

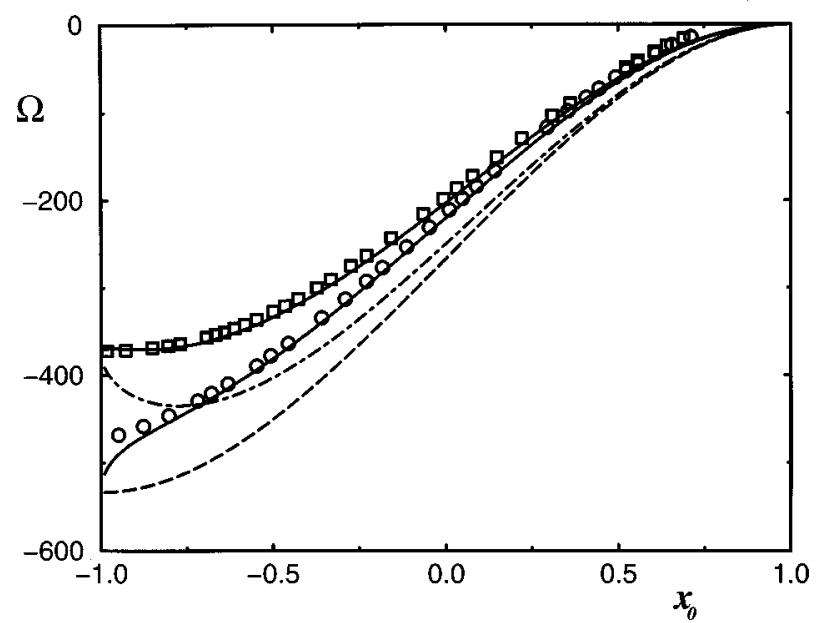

FIG. 2. Free energy, in arbitrary units, as a function of $x_{0}=\Delta \sigma / \sigma$ for fixed pressure difference $\Delta p / k=100$. The expansion parameter $(k / \sigma)^{1 / 2}=0.1$. Circles and squares are the results obtained by numerical solution of the profile for $\delta=0$ and $\delta=0.1$, respectively. The dashed and the dotted-dashed curve are $\Omega$ in Eq. (8) with $k=0$ for $\delta=0$ and $\delta=0.1$, respectively. The solid curves are the full free energy $\Omega$ in Eq. (8).

where $R_{p}=2 \sigma / \Delta p$ and $x_{0}=\Delta \sigma / \sigma$ are the radius and contact angle, respectively, to leading order in the expansion in $(k / \sigma)^{1 / 2} / R$ and $\delta / R$ (see Eqs. (4) and (5)).

The above calculation can also be carried out in the constant volume ensemble instead of the constant pressure ensemble. The result is

$$
\begin{aligned}
F= & \pi \sigma R_{V}^{2}\left(2+3 x_{0}-x_{0}^{3}\right)-4 \pi \delta \sigma R_{V}\left(1+x_{0}\right) \\
& -2 \pi \delta \sigma R_{V}\left(1-x_{0}^{2}\right)^{1 / 2} \arccos \left(x_{0}\right) \\
& +4 \pi R_{V}(k \sigma)^{1 / 2}\left(1-x_{0}^{2}\right)^{1 / 2}\left[2-2^{1 / 2}\left(1+x_{0}\right)^{1 / 2}\right],
\end{aligned}
$$

where $R_{V}=\left[3 V / \pi\left(2+3 x_{0}-x_{0}^{3}\right)\right]^{1 / 3}$ is the radius to leading order in the expansion in $(k / \sigma)^{1 / 2} / R$.

In order to test the accuracy of Eq. (8), we compare it to the exact free energy, found by solving the shape equations numerically, as a function of $x_{0}=\Delta \sigma / \sigma$. The result is shown in Fig. 2. Here we have chosen $(k / \sigma)^{1 / 2}=0.1$ in some arbitrary microscopic length unit, and fixed the pressure difference $\Delta p / k=100$ so that $R_{p}=2$, large compared to $(k / \sigma)^{1 / 2}$. The circles and squares in Fig. 2 are the numerically exact results for $\delta=0$ and $\delta=0.1$, respectively. The dashed curve $(\delta=0)$ and the dotted-dashed curve $(\delta=0.1)$ are the free energy $\Omega_{0}$ found by setting $k=0$ in Eq. (8). The solid curve is the full free energy $\Omega$ in Eq. (8) for both $\delta=0$ and $\delta=0.1$. As can be seen, it agrees well with the numerically obtained free energy when the contact angle is not too close to $x_{0}=-1\left(\theta=180^{\circ}\right)$; the complete wetting limit in which the vesicle completely spreads onto the solid substrate.

Finally we come back to droplet aggregation in microemulsions. With the formulas derived above we are able to calculate the free energy for the formation of dimers and construct the phase diagram for droplet-dimer coexistence. This is an important first step but for a full understanding of aggregation in microemulsions, entropy must be included (which we neglect since curvature energy is expected to be 


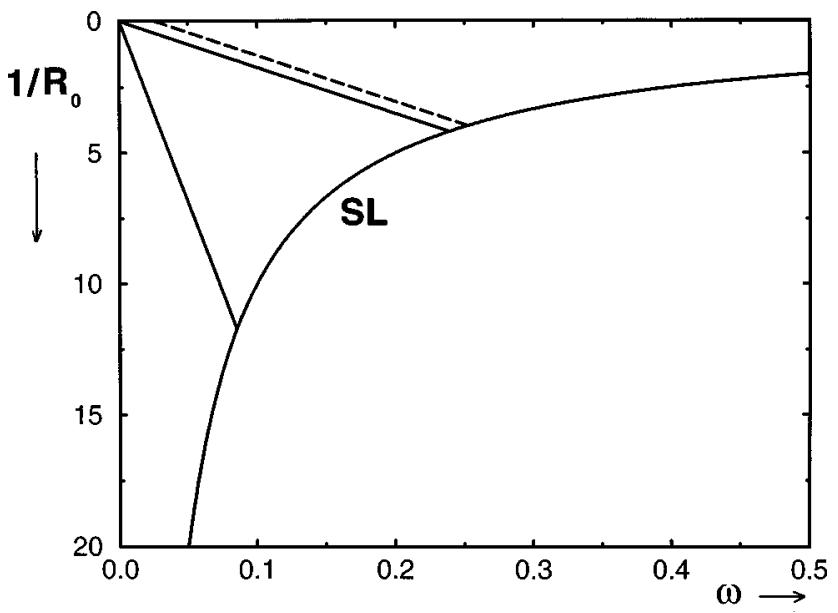

FIG. 3. Phase diagram for the dimerization transition in a microemulsion system as a function of $1 / R_{0}$ and $\omega \equiv V / A$. The solid curve SL is the solubilization limit beyond which the internal component is present as an excess phase. We have chosen $\sigma_{b} / \sigma=1,(k / \sigma)^{1 / 2}=0.1$ and two values for $\beta$. The solid curves denote the dimerization transition for $\beta=2.1$ (left) and $\beta=6$ (right) without rigidity. The dashed curve is the dimerization transition for $\beta=6$ with rigidity.

the dominant contribution to the free energy ${ }^{10,21}$ ), and the possible formation of higher aggregates needs to be considered.

For a given microemulsion the total volume and surface area of all the droplets is fixed by the total amount of the internal component (the component inside the microemulsion droplet) and surfactant, respectively. Considering the formation of dimers, let $N_{m}$ and $N_{d}$ denote the number of monomers and dimers with radius $R_{m}$ and $R_{d}$, respectively. Then $V_{\text {tot }}=N_{m} V_{m}+N_{d} V_{d}$ and $A_{\text {tot }}=N_{m} A_{m}+N_{d} A_{d}+N_{d}(\beta$ -2) $O_{d}$, where the parameter $\beta$ is the ratio of the number of surfactants per unit area in the bilayer to those in the monolayer. Realistically one expects this ratio to be close to 2 . The volume and surface area of the single droplet are simply given by $V_{m}=\frac{4}{3} \pi R_{m}^{3}$ and $A_{m}=4 \pi R_{m}^{2}$, whereas the volume of the dimer $V_{d}=(\pi / 3) R_{d}^{3}\left(2+3 x-x^{3}\right)$. The total surface area of the dimer $A_{d}$ and surface area of the flat part $O_{d}$ of the dimer are given by

$$
\begin{aligned}
A_{d}= & 2 \pi R_{d}^{2}\left(3+2 x-x^{2}\right)+8 \pi R_{d}(1-x)^{1 / 2}(k / \sigma)^{1 / 2} \\
& \times\left[(1+x)^{1 / 2}-2^{1 / 2}\right], \\
O_{d}= & \pi R_{d}^{2}\left(1-x^{2}\right)-2^{3 / 2} \pi R_{d}(1-x)^{1 / 2}(k / \sigma)^{1 / 2} .
\end{aligned}
$$

We now investigate the occurrence of a monomer to dimer transition by minimizing the curvature free energy with respect to $N_{m}, R_{m}, N_{d}, R_{d}$, and $x$, keeping $V_{\text {tot }}$ and $A_{\text {tot }}$ fixed. Details of the calculation will be presented elsewhere but typical results are shown in Fig. 3. Here the phase diagram is depicted as a function of the inverse radius of spontaneous curvature $1 / R_{0}$ (which is varied by changing the temperature) and the internal phase to surfactant ratio parameter $\omega$ $\equiv V_{\text {tot }} / A_{\text {tot }}$. The solid curve SL, defined by $1 / R_{0}=1 / 3 \omega$, is the solubilization limit beyond which $\left(\omega>\omega_{S L}\right)$ the microemulsion droplet phase coexists with an excess water or oil phase. ${ }^{9,19}$ It should be noted that Fig. 3 only displays the lower part of the one-phase region with the solubilization limit curve as the lower boundary. The upper part with the upper phase boundary above which two microemulsion phases form, usually described in terms of a liquid-gas transition, is not shown.

The two solid curves in Fig. 3 are the loci of the monomer-dimer transition for $\beta=2.1$ (left curve) and $\beta=6$ (right curve) without rigidity. The transition from monomers to dimers indeed occurs with decreasing $1 / R_{0}$ (increasing $\omega$ ). Keeping in mind the assumptions made in our calculation, this seems to prove that curvature energy is the driving force behind the attraction between droplets that ultimately may lead to phase separation. ${ }^{6,7}$ Since the monomer droplet radius $R_{m}=3 \omega$, dimerization occurs also with increasing droplet radius. This has indeed been observed by Huang et $a .^{22}$ in small angle neutron scattering experiments. The dimerization transition is continuous (second order) when $\beta<\beta_{c}=2$ $+\frac{4}{3} \sqrt{ } 3=4.30 \ldots$ and first order when $\beta>\beta_{c}$. The locus of continuous transitions is determined by

$$
\frac{1}{R_{0}}=\frac{3}{8} \frac{\omega}{k} \frac{\beta \sigma-\sigma_{b}}{\beta-2} .
$$

The presence of rigidity shifts the first order transition to lower $1 / R_{0}$ (higher $\omega$ ) as can be seen by the dashed curve in Fig. 3. To leading order in $k$, however, the presence of rigidity does not affect the location of the continuous transition.

In conclusion we have shown that the curvature energy of the surfactant layer accounts for the experimentally observed tendency of microemulsion droplets to form dimers (and eventually larger aggregates) in the direction of vanishing spontaneous curvature. This phenomenon could not be sufficiently explained by liquid state theories (like the sticky hard sphere model) since aggregation is observed both with increasing and decreasing temperature, while liquid state theories assume aggregation to be driven by entropy thus only occurring in one temperature direction. Since curvature energy is expected to be the dominant contribution to the free energy (see, for example, Refs. 10 and 21), the point we have made concerning the formation of dimers in the direction of vanishing spontaneous curvature will not be significantly affected by the inclusion of entropy.

The authors wish to express their gratitude to Dirk Jan Bukman for his help with deriving Eq. (3). The research of E.M.B. has been made possible by a fellowship of the Royal Netherlands Academy of Arts and Sciences. The work of W.F.C.S. has been supported by the Netherlands Foundation for Chemical Research (SON) in collaboration with the Netherlands Technology Foundation (STW).

\footnotetext{
${ }^{1}$ W. Helfrich, Z. Naturforsch. C 28, 693 (1973).

${ }^{2}$ For reviews, see Micelles, Membranes, Microemulsions, and Monolayers, edited by W.M. Gelbart, A. Ben-Shaul, and D. Roux (Springer, New York, 1994); Statistical Mechanics of Membranes and Surfaces, edited by D. Nelson, T. Piran, and S. Weinberg (World Scientific, Singapore, 1988); M. Wortis, U. Seifert, K. Berndl, B. Fourcade, M. Rao, and R. Zia, in Dynamical Phenomena at Interfaces, Surfaces and Membranes, edited by D. Beysens, N. Boccara, and G. Forgacs (Nova Science, New York, 1993).

${ }^{3}$ E. M. Blokhuis and D. Bedeaux, Physica A 164, 515 (1990); 184, 42
} 
(1992); E. M. Blokhuis and D. Bedeaux, Heterog. Chem. Rev. 1, 55 (1994).

${ }^{4}$ U. Seifert and R. Lipowsky, Langmuir 42, 4768 (1990).

${ }^{5}$ U. Seifert, Z. Phys. B 97, 299 (1995); R. Lipowsky and U. Seifert, Langmuir 7, 1867 (1991); U. Seifert, Phys. Rev. Lett. 74, 5060 (1995).

${ }^{6} \mathrm{~S}$. H. Chen, C. Y. Ku, J. Rouch, P. Tartiaglia, C. Cametti, and J. Samseth, J. Phys. IV 3, 143 (1993).

${ }^{7}$ W. F. C. Sager and E. M. Blokhuis, Prog. Colloid Polym. Sci. 110, 258 (1998).

${ }^{8}$ H. J. Deuling and W. Helfrich, J. Phys. (Paris) 37, 1335 (1976); L. Miao, B. Fourcade, M. Rao, M. Wortis, and R. Zia, Phys. Rev. A 43, 6843 (1991); U. Seifert, Adv. Phys. 46, 13 (1997).

${ }^{9}$ J. S. Huang, S. T. Milner, B. Farago, and D. Richter, Phys. Rev. Lett. 59, 2600 (1987); M. Borkovec and H.-F. Eicke, Chem. Phys. Lett. 157, 457 (1989); H. Kellay and J. Meunier, J. Phys.: Condens. Matter 8, A49 (1996); U. Olsson and H. Wennerström, Adv. Colloid Interface Sci. 49, 113 (1994).

${ }^{10}$ See also S. A. Safran, Statistical Thermodynamics of Surfaces, Interfaces, and Membranes (Addison-Wesley, Reading, 1994), and references therein.

${ }^{11}$ D. Chatenay, W. Urbach, A. M. Cazabat, and D. Langevin, Phys. Rev. Lett. 54, 2253 (1985); S.-H. Chen, S.-L. Chang, and R. Strey, J. Chem.
Phys. 93, 1907 (1990); G. J. M. Koper, W. F. C. Sager, J. Smeets, and D. Bedeaux, J. Phys. Chem. 99, 13291 (1995).

${ }^{12}$ C. Robertus, J. G. H. Joosten, and Y. K. Levine, Phys. Rev. A 42, 4280 (1990).

${ }^{13}$ T. N. Zemb, S. T. Hyde, P.-J. Derian, I. S. Barnes, and B. W. Ninham, J. Phys. Chem. 91, 3814 (1987).

${ }^{14}$ W. Wintz, H.-G. Döbereiner, and U. Seifert, Europhys. Lett. 33, 403 (1996).

${ }^{15}$ R. Barziv, R. Menes, E. Moses, and S. A. Safran, Phys. Rev. Lett. 75, 3356 (1995).

${ }^{16}$ R. C. Tolman, J. Chem. Phys. 17, 333 (1949).

${ }^{17}$ S. Giasson, T. Kuhl, and J. N. Israelachvili, Langmuir 14, 891 (1998); R. Aveyard, B. P. Binks, W.-G. Cho, L. R. Fisher, P. D. I. Fletcher, and F. Klinkhammer, ibid. 12, 6561 (1996).

${ }^{18}$ P. D. I. Fletcher and D. N. Petsev, J. Chem. Soc., Faraday Trans. 93, 1983 (1997).

${ }^{19}$ U. Olsson and P. Schurtenberger, Langmuir 9, 3389 (1993).

${ }^{20}$ J. S. Rowlinson and B. Widom, Molecular Theory of Capillarity (Clarendon, Oxford, 1982).

${ }^{21}$ P. G. de Gennes and C. Taupin, J. Phys. Chem. 86, 2294 (1982).

${ }^{22}$ J. S. Huang, S. A. Safran, M. W. Kim, G. S. Grest, M. Kotlarchyk, and N. Quirke, Phys. Rev. Lett. 53, 592 (1984). 